\title{
Optical signature of bipolaron in monolayer transition metal dichalcogenides: all coupling approach
}

\author{
Nguepnang J.V², Kenfack-Sadem C. ${ }^{1,4, *}$, Kenfack-Jiotsa A. ${ }^{3}$, M.F.C Fobasso ${ }^{1}$, Y. \\ Sun $^{5, *}$
}

1. Laboratory of Condensed Matter-Electronics and Signal Processing (LAMACET), Department of Physics, University of Dschang, P.O.Box 67, Dschang, Cameroon.

2. Laboratoire de Mécanique, Matériaux et Structures, Département de Physique, Université de Yaoundé 1, BP 812, Yaoundé, Cameroon

3. Département de Physique, Ecole Normale Supérieure, Université de Yaoundé 1, BP 42, Yaoundé, Cameroon.

4. International Chair in Mathematical Physics and Applications (ICMPA-UNESCO Chair), University of Abomey-Calavi, 072 B.P. 50, Cotonou, Benin

5. Institute of Condensed Matter Physics, College of Mathematics and Physics, Inner Mongolia University for Nationalities, Tongliao, China.

*Email: christian.kenfack@univ-dschang.org

*Email :sy19851009@126.com ;sunyong@imun.edu.cn

\begin{abstract}
We studied the optical signature of bipolaron and its effects on the bandgap modulation in the single-layer Transition Metal Dichalcogenides (TMDs) under magnetic field. Using the Huybrecht method, we derived the ground state energies in the modified zero Landau levels for all Fröhlich coupling constants. We take into account both intrinsic longitudinal optical phonon modes and surface optical phonon modes induced by the polar substrate. We observed that the higher the coupling strength, the stronger is the magnetic field effect. The highest amplitude of the bandgap modulation is obtained for the $\mathrm{MoS}_{2}$ monolayer and the lowest with the $\mathrm{WSe}_{2}$ monolayer. We also found that the bipolaron is stable in TMDs. It is seen that the optical absorption presents the threshold values and respectively increases for $\mathrm{WSe}_{2}$, $\mathrm{MoSe}_{2}, \mathrm{WS}_{2}$ and $\mathrm{MoS}_{2}$.
\end{abstract}

Keywords: Bipolaron, Bandgap, Magnetic field,TMDs, electron-phonon interaction

\section{Introduction}

Transition metal dichalcogenides belong to the family of lamellar materials of formula $\mathrm{MX}_{2}$. $\mathrm{M}$ is a transition metal of group IV, V or VI of the periodic classification table and $\mathrm{X}$ is a chalcogen. Electronically, it covers a wide range of properties from insulator to semiconductor and to the metal [1]. Two-dimensional (2D) TMDs is a new generation of thin atomic material with special physical properties. Today, these materials are the focus of much scientific research $[2,3]$. Compared to graphene, the direct bandgap of TMDs has been reduced, with potential 
applications for high electron mobility transistors and light-emitting diodes [4]. Due to the ability to control the size of the 2D TMDs bandgap, the TMDs bandgap has become one of the most important studies in semiconductor physics [5,6].

Polaron is an entity discovered in 1933 by Landau [7], it is use to describe interaction between free charges carriers and induced polarization coming from electron (hole)-atome coupling in solid material. This quasiparticle is characterized by some properties as effective mass, energy mobility and absorption coefficient, etc. These properties strongly depend on the interaction between charges carriers, crystal lattice and the frequence of charges carriers [8,9]. Depending on the way the size of lattice distorsion is, comparing to lattice constant, they are two types of polaron: large polaron and small polaron [10-12]. The bipolaron arrives when two identical polaron interact in the crystal lattice. Bipolaron formation is influenced by the difference between the coulombic repulsion of chages carriers and theirs attraction with lattice.

Many theoretical works investigated the polaron in 2D structure through the chemical composition [13], the geometrical shapes of the material [14] and the lattice constant $[15,16]$. Other theoretical works exhibit high bandgap renormalization [17,18]. Experimental work shows that polar substrates are important in the study of the physical properties of TMDs $[19,20]$ and also highlights the importance of the Van der Waals interaction $[18,21,22]$ in TMDs. Based on these results, it appears that the environment may affect the TMD band gap. In a recent paper [23], the study of the modulated bandgap in 2D single-layer TMDs derived from carrier-optic phonon coupling in the Fröhlich model showed that the bandgap magnitude can be modulated in the range of $100-500 \mathrm{meV}$ by changing different polar substrates and by varying the internal distance between the TMDs and the polar substrates. These studies only considered the weak coupling regime. Nevertheless, it is necessary to explore all coupling regimes while study bipolaron in order to better understand the modulation bandwidth and the stability in TMDs. Optical conductivity and infrared absorption characterized by the optical absorption coefficient of the Fröhlich polaron have been studied in polar semiconductors and ion crystals [24-26]. Experimental and theoretical works have been used to derive the optical absorption [27-29]. Recently, Li and Wang studied the optical absorption coefficient of the Fröhlich polaron in single-layer TMDs taking in account both LO and SO phonons [30]. They derived the optical absorptions using the Devreese-Huybrechts-Lemmens model in the low temperature limit for the weak coupling between the electron and the phonon. However, the optical absorption of bipolaron in TMD, to our awareness, has not been sufficiently studied. 


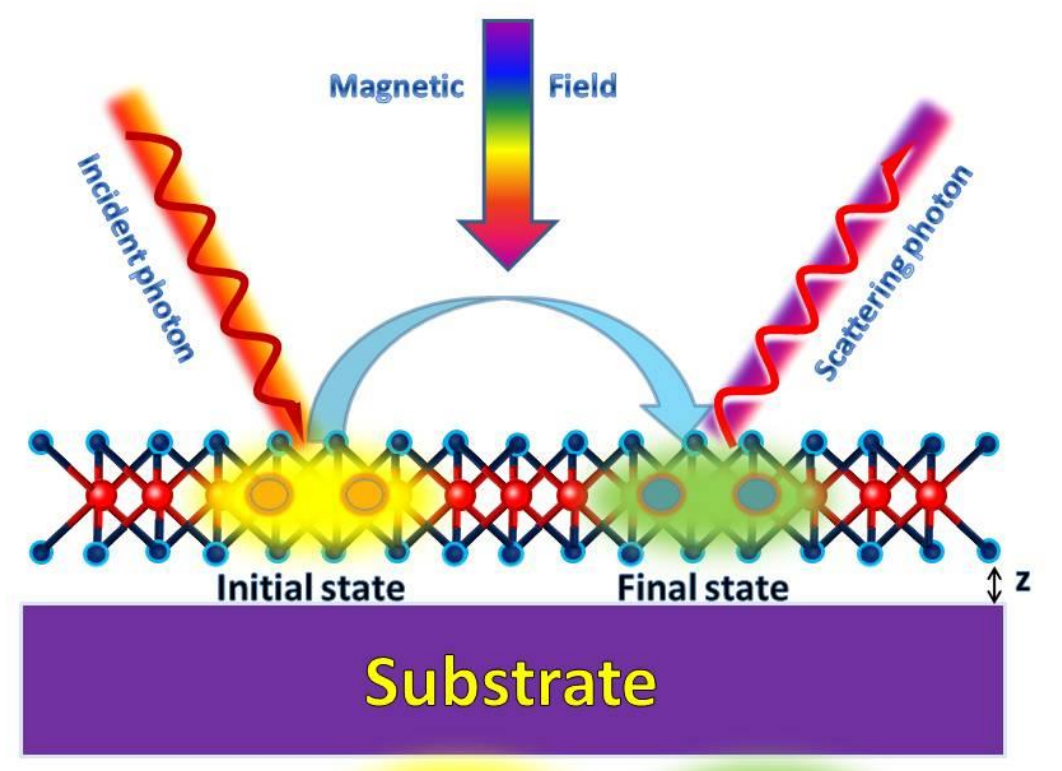

$\mathrm{X} \odot \mathrm{M}$

Bipolaron

FIG. 1. Sketch of the optical absorption of the bipolaron in the monolayer TMDS

In this work, we first present the effect of the bipolaron for all coupling regime on the modulated bandgap in a two-dimensional TMD quantum dot supported by a polar substrate in the presence of an external magnetic field in the third direction. We study the optical absorption of bipolaron in TMDs for all coupling regime. The optical phononic modes are strongly coupled with the carriers in 2D TMDs [31], which leads to the formation of the polar state [32]. We have taken into account the intrinsic longitudinal optical phononic modes (LO) and the surface optical phononic modes (SO) induced by the polar substrate.

We organized the article as follows: in section 2, we present the conceptual model and the calculations. The results are given and discussed in section 3 , and in section 4 , we conclude.

\section{Model and calculations}

\section{A. Ground state ennergies}

Let us consider a monolayer of TMDs situates on a polar substrate in presence of uniform magnetic field applied on the third dimensions in a quantum dot (fig. 1.). The total Hamiltonian of bipolaron can be written in this form

$$
H_{b p}=H_{e}+H_{p h}+H_{e-p h}+u(r)+U\left(\left|r_{1}-r_{2}\right|\right)
$$

where $H_{e}$ describes the energy of the electron defined as: 
$H_{e}=\gamma V_{F} \sum_{i=1,2}\left(\sigma_{1}\left(p_{i x}-2 e A_{x}\right)+\sigma_{2}\left(p_{i y}-2 e A_{y}\right)+\sigma_{3}\left(p_{i z}-2 e A_{z}\right)+\sigma_{3} G\right)$

with $\sigma_{1}, \sigma_{2}, \sigma_{3}$ the Pauli matrices, $V_{F}$ the Fermi velocity, $2 G$ the bandgap, $(\gamma= \pm 1)$ for electrons and holes respectively and $A$ is the potential.

The second term $H_{p h}$ stands for the phonon energies including SO and LO modes defined as:

$$
H_{p h}=\sum_{k, v} \hbar \omega_{v} a_{k}^{+} a_{k}
$$

where $a_{k}^{+}, a_{k}$ are respectively creation and annihilation operators for the phonon with $k$ being wave vector where $\omega_{v}$ is the frequency of the phonons.

The third term $H_{e-p h}$ is the Hamiltonian of interaction between electron and phonon

$$
H_{e-p h}=\sum_{k, v} M_{k, v}\left(a_{-k}^{+}+a_{k}\right) \exp (i k r)
$$

where $M_{k, v}$ is the coupling element of Fröhlich [23].

The term $u(r)$ is the confinement potential [33] in the quantum dot

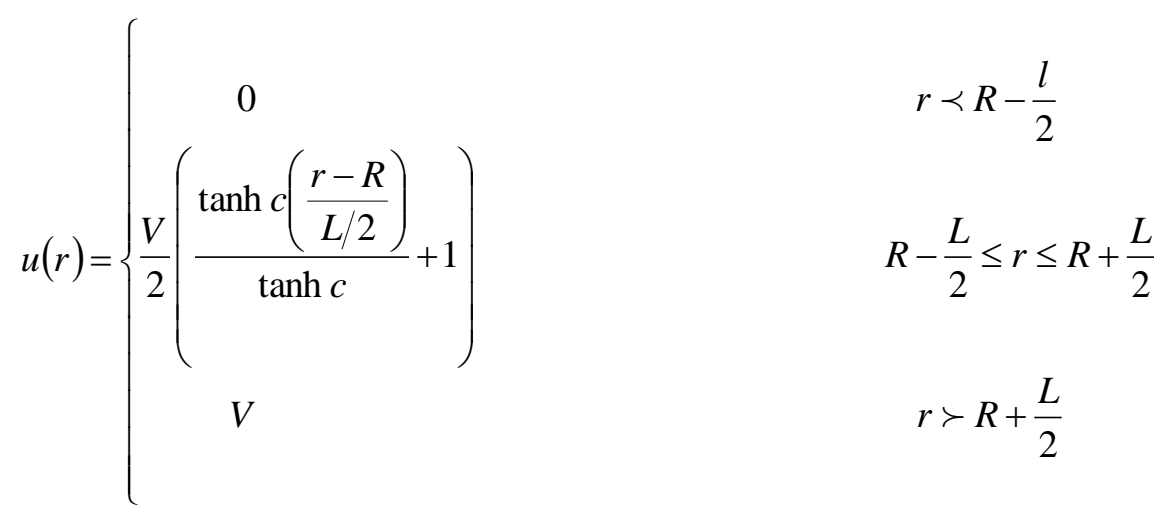

whereas $R$ and $V$ are respectively the length and the depth of the quantum dot and $L$ is the smoothness of quantum dot. $C$ is a constant. The last term of Eq. (1). is the coulomb interaction potential between the two electrons. Since the bipolaron is a composite particle, it is convenient to introduce the center of mass and relative coordinate and momenta,

$$
P_{x}=p_{1 x}+p_{2 x}, P_{y}=p_{1 y}+p_{2 y}, P_{z}=p_{1 z}+p_{2 z}, r=r_{1}-r_{2} \quad R_{1}=\frac{r_{1}+r_{2}}{2} \text {. The }
$$

Hamiltonian in Eq. (1). can be rewritten after averaging over a relative wavefunction $\psi(r)$ as :

$$
H_{b p}=\gamma V_{F} \sum_{i=1,2}\left(\sigma_{1} \pi_{i x}+\sigma_{2} \pi_{i y}+\sigma_{3} \pi_{i z}+\sigma_{3} G\right)+\sum_{k, v} B_{k, v}\left(a_{-k}^{+}+a_{k}\right) \exp \left(i k R_{1}\right)+\sum_{k, v} \hbar \omega_{v} a_{k}^{+} a_{k}+E_{r}+u(r)
$$


With $B_{k, v}=2 M_{k v}\left\langle\cos \left(\frac{k r}{2}\right)\right\rangle$ and $E_{r}=\left\langle\frac{U}{r}\right\rangle$.

$\langle.$.$\rangle denoting an averaging over the wavefunction \psi_{0}^{o}(r)=c \exp \left(-\frac{b r^{2}}{4}\right)$ as used in reference [34].

The Huybrecht method is applied by using the two following unitary transformations :

$$
\left\{\begin{array}{l}
u_{1}=\exp \left(-i \alpha \sum_{k} k r a_{k}^{+} a_{k}\right) \\
u_{2}=\exp \left(\sum_{k}\left(f_{k} a_{k}^{+}+f_{k}^{*} a_{k}\right)\right)
\end{array}\right.
$$

Where $\alpha$ is parameter, $f_{k}$ and $f_{k}^{*}$ are the variationnal parameter. Performing the two Huybrechts unitary tranformation to the bipolaron's Hamiltonian, one gets :

$$
H_{b p}^{\prime}=u_{2}^{-1} u_{1}^{-1} H_{b p} u_{1} u_{2}
$$

then

$$
\begin{aligned}
& H_{b p}^{\prime}=V_{F}\left[\sigma_{i} \pi_{i}-\sum_{k, i} \hbar a \sigma_{i} k_{i}\left(a_{k}^{+}+f_{k}^{*}\right)\left(a_{k}+f_{k}\right)+\sigma_{3} G\right]+E_{r}+u(r)+\sum_{k, v} \hbar \omega_{v}\left(a_{k}^{+}+f_{k}^{*}\right)\left(a_{k}+f_{k}\right)+ \\
& \sum_{k, v} B_{k, v}\left(a_{k}^{+}+f_{k}^{*}\right)\left(a_{k}+f_{k}\right) \exp \left[i k R_{1}(1-\alpha)\right]
\end{aligned}
$$

In order to investigate the ground state energy, we will replace into Eq. (8). $\boldsymbol{P}_{\boldsymbol{i}}$ and $r_{i}$ by the expression given by $\mathrm{P}_{i}=\left(\frac{m \hbar \lambda}{2}\right)^{1 / 2}\left(b_{j}^{+}+b_{j}\right)$ and $r_{i}=\left(\frac{\hbar}{2 m \lambda}\right)^{1 / 2}\left(b_{j}-b_{j}^{+}\right)$where $\lambda=\sqrt{e B / 2 \hbar}$ is the magnetic confinement length, $b_{j}^{+}$the creation and $b_{j}$ the annihilation operators are introduced for momentum and positon of electron, subscript $i$ denote $x, y, z$.

Then minimizing by the zero phonon $\left|\psi_{0}\right\rangle_{e}|0\rangle_{p h}=\left(\begin{array}{c}0 \\ |0\rangle_{e}\end{array}\right)|0\rangle_{p h}$ we obtain:

$$
E_{b p 0+}=V_{F}\left[\sigma_{3} G-\sum_{k, i} \hbar a k_{i} \sigma_{i} f_{k}^{*} f_{k}\right]+\sum_{k, v} \hbar \omega_{v} f_{k}^{*} f_{k}+\sum_{k, v} B_{k, v}\left(f_{k}^{*}+f_{k}\right) \exp \left[-(1-\alpha)^{2} \frac{\hbar}{2 m \lambda} k^{2}\right]+U+E_{r}
$$


with

$$
\begin{gathered}
f_{k}=-\frac{2 M_{k, v} \exp \left[-(1-\alpha)^{2} \frac{\hbar}{2 m \lambda} k^{2}\right] \exp \left(-\frac{k^{2}}{2 b}\right)}{V_{F} \alpha \hbar k+\hbar \omega_{v}}, \\
f_{k}^{*}=-\frac{2 M_{k, v}^{*} \exp \left[-(1-\alpha)^{2} \frac{\hbar}{2 m \lambda} k^{2}\right] \exp \left(-\frac{k^{2}}{2 b}\right)}{V_{F} \alpha \hbar k+\hbar \omega_{v}}
\end{gathered}
$$

And

$$
\begin{aligned}
& \int \quad r \prec R-\frac{l}{2} \\
& U=\left\{\frac{V}{2}\left(\frac{\tanh c\left(\frac{-2 R}{L}\right)}{\tanh c}+1\right) \quad R-\frac{L}{2} \leq r \leq R+\frac{L}{2} .\right. \\
& \text { V } \\
& r \succ R+\frac{L}{2}
\end{aligned}
$$

Applying the wavefunction $\psi_{0}^{o}(r)=c \exp \left(-\frac{b r^{2}}{4}\right)$ we obtain

$$
B_{k, \nu}=2 M_{k \nu} \exp \left(-\frac{k^{2}}{2 b}\right) \text { and } E_{r}=4 \pi^{2}\left(\frac{b}{2 \pi}\right)^{1 / 2} \frac{e^{2}}{\varepsilon_{\infty} b} \text {. }
$$

Then we obtain the bipolaron energy :

$$
\begin{aligned}
& E_{b p 0++}=-\sum_{k, \nu} 4 \alpha \sigma_{1} \hbar V_{F} k_{x} \frac{M_{k, \nu} M_{k, \nu}^{*} \exp \left[-(1-\alpha)^{2} \frac{\hbar}{m \lambda} k^{2}\right] \exp \left(-\frac{k^{2}}{b}\right)}{\left(V_{F} \alpha \hbar k+\hbar \omega_{v}\right)^{2}}-\sum_{k, \nu} 4 \alpha \sigma_{2} \hbar V_{F} k_{y} \frac{M_{k, \nu} M_{k, \nu}^{*} \exp \left[-(1-\alpha)^{2} \frac{\hbar}{m \lambda} k^{2}\right] \exp \left(-\frac{k^{2}}{b}\right)}{\left(V_{F} \alpha \hbar k+\hbar \omega_{v}\right)^{2}} \\
& -\sum_{k, \nu} 4 \alpha \sigma_{3} \hbar V_{F} k_{z} \frac{M_{k, \nu} M_{k, \nu}^{*} \exp \left[-(1-\alpha)^{2} \frac{\hbar}{m \lambda} k^{2}\right] \exp \left(-\frac{k^{2}}{b}\right)}{\left(V_{F} \alpha \hbar k+\hbar \omega_{v}\right)^{2}}+\sum_{k, \nu} 4 \hbar \omega_{\nu} \frac{M_{k, \nu} M_{k, \nu}^{*} \exp \left[-(1-\alpha)^{2} \frac{\hbar}{m \lambda} k^{2}\right] \exp \left(-\frac{k^{2}}{b}\right)}{\left(V_{F} \alpha \hbar k+\hbar \omega_{v}\right)^{2}}+U \\
& +4 \pi^{2}\left(\frac{b}{2 \pi}\right)^{1 / 2} \frac{e^{2}}{\varepsilon_{\infty} b}+\sum_{k, \nu} 8 \frac{M_{k, \nu} M_{k, \nu}^{*} \exp \left[-(1-\alpha)^{2} \frac{\hbar}{2 m \lambda} k^{2}\right] \exp \left(-\frac{k^{2}}{2 b}\right)}{\left(V_{F} \alpha \hbar k+\hbar \omega_{\nu}\right)}+\sigma_{3} V_{F} G
\end{aligned}
$$

Where $E_{b p 0+}$ is the modified zero Landau level due to the bipolaron effect for the electron. Following the same rules, $E_{b p 0-}$ is for the case of the hole and can also be obtained in the top of the valence band. Finally, the eigenvalues of the energies in the zero Landau Levels can be written as : 


$$
\begin{aligned}
& E_{b p 0 \pm \pm}= \pm \sqrt{\left(V_{F} G\right)^{2}+\left(4 \hbar V_{F} \alpha \sum_{k ; \nu} k \frac{\left.M_{k, v}^{2} \exp \left[-(1-\alpha)^{2} \frac{\hbar}{m \lambda} k^{2}\right] \exp \left(-\frac{k^{2}}{b}\right)\right)^{2}}{\left(V_{F} \alpha \hbar k+\hbar \omega_{v}\right)^{2}} \pm U \pm 4 \pi^{2}\left(\frac{b}{2 \pi}\right)^{1 / 2} \frac{e^{2}}{\varepsilon_{\infty} b}\right.} \\
& \pm 4 \sum_{k, \nu} \hbar \omega_{v} \frac{M_{k, \nu}^{2} \exp \left[-(1-\alpha)^{2} \frac{\hbar}{m \lambda} k^{2}\right] \exp \left(-\frac{k^{2}}{b}\right)}{\left(V_{F} \alpha \hbar k+\hbar \omega_{v}\right)^{2}} \mp 8 \sum_{k, \nu} \frac{M_{k, v}^{2} \exp \left[-(1-\alpha)^{2} \frac{\hbar}{2 m \lambda} k^{2}\right] \exp \left(-\frac{k^{2}}{2 b}\right)}{\left(V_{F} \alpha \hbar k+\hbar \omega_{v}\right)}
\end{aligned}
$$

Surface optical phonons strongly coupling with carriers in monolayer TMDs are described by the third term in Eq. (1). with the coupling element [35-36]:

$$
M_{k, s o}=\sqrt{\frac{e^{2} \eta \hbar \omega_{s o, v}}{2 A \varepsilon_{0} k}} \exp \left(-k z_{0}\right)
$$

$\eta$ is the polarizability of the substrate, $\varepsilon_{0}$ is low frequency dielectric constant, $z_{0}$ is the internal distance between the TMDs and substrate. $\hbar \omega_{S O, v}$ is the energy of SO phonon with two branches $v=1,2$. Carriers coupled with intrinsic longitudinal optical phonon coupling in monolayer TMDs have been studied extensively [38-40]. The coupling element in Fröhlich model is given by

$$
M_{k, L O}=\sqrt{\frac{e^{2} \eta_{0} L_{m} \hbar \omega_{L O}}{2 A \varepsilon_{0}}} \operatorname{erfc}\left(\frac{k \sigma}{2}\right)
$$

Where $L_{m}$ is the atomic thickness of the monolayer, $\eta_{0}$ is the intrinsic polarizability of material, $\hbar \omega_{L O}$ represents the energy of LO phonon and erfc is the complementary error function. $\sigma$ denotes the effective width of the electronic Bloch states reflecting the confinement effect between LO phonons and carriers in 2D materials [38,40]. Replacing Eqs. (14a). or (14b). we obtain the following results for different coupling regime and for SO and LO phonons :

The energies of SO and LO in the weak coupling $(\alpha=1)$ are respectively :

$$
\begin{aligned}
& \left(E_{b p 0 \pm}\right)_{S O}= \pm \sqrt{\left(V_{F} G\right)^{2}+\left(\frac{\hbar V_{F} e^{2} \eta}{\pi \varepsilon_{0}} \sum_{v=1,2} \hbar \omega_{S O, \nu} \int_{0}^{K_{c}} k d k \frac{\left.\exp \left(-2 k z_{0}\right) \exp \left(-\frac{k^{2}}{b}\right)\right)^{2}}{\left(V_{F} \hbar k+\hbar \omega_{S O, \nu}\right)^{2}} \pm U \pm 4 \pi^{2}\left(\frac{b}{2 \pi}\right)^{1 / 2} \frac{e^{2}}{\varepsilon_{\infty} b}\right.} \\
& \pm \frac{e^{2} \eta}{\pi \varepsilon_{0}} \sum_{\nu=1,2}\left(\hbar \omega_{S O, \nu}\right)^{2} \int_{0}^{K_{c}} d k \frac{\exp \left(-2 k z_{0}\right) \exp \left(-\frac{k^{2}}{b}\right)}{\left(V_{F} \hbar k+\hbar \omega_{S O, \nu}\right)^{2}} \mp 2 \frac{e^{2} \eta}{\pi \varepsilon_{0}} \sum_{\nu=1,2} \hbar \omega_{S O, \nu} \int_{0}^{K_{c}} d k \frac{\exp \left(-2 k z_{0}\right) \exp \left(-\frac{k^{2}}{2 b}\right)}{\left(V_{F} \hbar k+\hbar \omega_{S O, \nu}\right)^{2}}
\end{aligned}
$$

And 


$$
\begin{aligned}
& \left(E_{b p 0 \pm}\right)_{L O}= \pm \sqrt{\left(V_{F} G\right)^{2}+\left(\frac{\hbar V_{F} e^{2} \eta_{0} L_{m} \hbar \omega_{L O}}{\pi \varepsilon_{0}} \int_{0}^{K_{c}} k^{2} d k \frac{\left.\left(\operatorname{erfc}\left[\frac{k \sigma}{2}\right]\right)^{2} \exp \left(-\frac{k^{2}}{b}\right)\right)^{2}}{\left(V_{F} \hbar k+\hbar \omega_{L O}\right)^{2}} \pm U \pm 4 \pi^{2}\left(\frac{b}{2 \pi}\right)^{1 / 2} \frac{e^{2}}{\varepsilon_{\infty} b}\right.} \\
& \pm \frac{e^{2} \eta_{0} L_{m}\left(\hbar \omega_{L O}\right)^{2}}{\pi \varepsilon_{0}} \int_{0}^{K_{c}} k d k \frac{\left(\operatorname{erfc}\left[\frac{k \sigma}{2}\right]\right)^{2} \exp \left(-\frac{k^{2}}{b}\right)}{\left(V_{F} \hbar k+\hbar \omega_{L O}\right)^{2}} \mp 2 \frac{e^{2} \eta_{0} L_{m} \hbar \omega_{L O}}{\pi \varepsilon_{0}} \int_{0}^{K_{c}} k d k \frac{\left(\operatorname{erfc}\left[\frac{k \sigma}{2}\right]\right)^{2} \exp \left(-\frac{k^{2}}{2 b}\right)}{\left(V_{F} \hbar k+\hbar \omega_{L O}\right)}
\end{aligned}
$$

The energies of SO and LO bipolaron in the intermediate coupling $(\mathrm{O} \prec \alpha \prec 1)$ are respectively :

$$
\begin{aligned}
& \left(E_{b p 0 \pm}\right)_{S O}= \pm \sqrt{\left(V_{F} G\right)^{2}+\left(\frac{\hbar V_{F} e^{2} \alpha \eta}{\pi \varepsilon_{0}} \sum_{\nu=1,2} \hbar \omega_{S O, \nu} \int_{0}^{K_{c}} k d k \frac{\left.\exp \left(-2 k z_{0}\right) \exp \left[-(1-\alpha)^{2} \frac{\hbar}{m \lambda} k^{2}\right] \exp \left(-\frac{k^{2}}{b}\right)\right)^{2}}{\left(V_{F} \hbar \alpha k+\hbar \omega_{S O, \nu}\right)^{2}}\right)^{2}} \\
& \pm U \pm 4 \pi^{2}\left(\frac{b}{2 \pi}\right)^{1 / 2} \frac{e^{2}}{\varepsilon_{\infty} b} \pm \frac{e^{2} \eta}{\pi \varepsilon_{0}} \sum_{\nu=1,2}\left(\hbar \omega_{S O, \nu}\right)^{2} \int_{0}^{K_{c}} d k \frac{\exp \left(-2 k z_{0}\right) \exp \left[-(1-\alpha)^{2} \frac{\hbar}{m \lambda} k^{2}\right] \exp \left(-\frac{k^{2}}{b}\right)}{\left(V_{F} \hbar \alpha k+\hbar \omega_{S O, \nu}\right)^{2}} \mp \\
& 2 \frac{e^{2} \eta}{\pi \varepsilon_{0}} \sum_{\nu=1,2} \hbar \omega_{S O, \nu} \int_{0}^{K_{c}} d k \frac{\exp \left(-2 k z_{0}\right) \exp \left[-(1-\alpha)^{2} \frac{\hbar}{2 m \lambda} k^{2}\right] \exp \left(-\frac{k^{2}}{2 b}\right)}{\left(V_{F} \hbar \alpha k+\hbar \omega_{S O, \nu}\right)^{2}}
\end{aligned}
$$

and

$$
\begin{aligned}
& \left(E_{b p O \pm}\right)_{L O}= \pm \sqrt{\left(V_{F} G\right)^{2}+\left(\frac{\hbar \alpha V_{F} e^{2} \eta_{0} L_{m} \hbar \omega_{L O}}{\pi \varepsilon_{0}} \int_{0}^{K_{f}} k^{2} d k \frac{\left.\left(\operatorname{erfc}\left[\frac{k \sigma}{2}\right]\right)^{2} \exp \left[-(1-\alpha)^{2} \frac{\hbar}{m \lambda} k^{2}\right] \exp \left(-\frac{k^{2}}{b}\right)\right)^{2}}{\left(V_{F} \hbar \alpha k+\hbar \omega_{L O}\right)^{2}} \pm U \pm 4 \pi^{2}\left(\frac{b}{2 \pi}\right)^{1 / 2} \frac{e^{2}}{\varepsilon_{\infty} b}\right.} \\
& \pm \frac{e^{2} \eta_{0} L_{m}\left(\hbar \omega_{L O}\right)^{2}}{\pi \varepsilon_{0}} \int_{0}^{K_{f}} k d k \frac{\left(\operatorname{erf}\left[\frac{k \sigma}{2}\right]\right)^{2} \exp \left[-(1-\alpha)^{2} \frac{\hbar}{m \lambda} k^{2}\right] \exp \left(-\frac{k^{2}}{b}\right)}{\left(V_{F} \hbar \alpha k+\hbar \omega_{L O}\right)^{2}} \mp 2 \frac{e^{2} \eta_{0} L_{m} \hbar \omega_{L O}}{\pi \varepsilon_{0}} \int_{0}^{K_{f}} k d k \frac{\left(\operatorname{erfc}\left[\frac{k \sigma}{2}\right]\right)^{2} \exp \left[-(1-\alpha)^{2} \frac{\hbar}{2 m \lambda} k^{2}\right] \exp \left(-\frac{k^{2}}{2 b}\right)}{\left(V_{F} \hbar \alpha k+\hbar \omega_{L O}\right)}
\end{aligned}
$$

The energies of SO and LO bipolaron in the strong coupling $(\alpha=0)$ are respectively :

$$
\begin{aligned}
& \left(E_{b p 0 \pm}\right)_{S O}= \pm V_{F} G \pm \frac{e^{2} \eta}{\pi \varepsilon_{0}} \int_{0}^{K_{c}} \exp \left(-2 k z_{0}\right) \exp \left[-\frac{\hbar}{m \lambda} k^{2}\right] \exp \left(-\frac{k^{2}}{b}\right) d k \\
& \mp 2 \frac{e^{2} \eta}{\pi \varepsilon_{0}} \int_{0}^{K_{c}} \exp \left(-2 k z_{0}\right) \exp \left[-\frac{\hbar}{2 m \lambda} k^{2}\right] \exp \left(-\frac{k^{2}}{2 b}\right) d k \pm U \pm 4 \pi^{2}\left(\frac{b}{2 \pi}\right)^{1 / 2} \frac{e^{2}}{\varepsilon_{\infty} b}
\end{aligned}
$$

and 


$$
\begin{aligned}
& \left(E_{b p 0 \pm}\right)_{L O}= \pm V_{F} G \pm \frac{e^{2} \eta_{0} L_{m}}{\pi \varepsilon_{0}} \int_{0}^{K_{c}} k\left(\operatorname{erfc}\left[\frac{k \sigma}{2}\right]\right)^{2} \exp \left[-\frac{\hbar}{m \lambda} k^{2}\right] \exp \left(-\frac{k^{2}}{b}\right) d k \\
& \mp 2 \frac{e^{2} \eta_{0} L_{m}}{\pi \varepsilon_{0}} \int_{0}^{K_{c}} k\left(\operatorname{erfc}\left[\frac{k \sigma}{2}\right]\right)^{2} \exp \left[-\frac{\hbar}{2 m \lambda} k^{2}\right] \exp \left(-\frac{k^{2}}{2 b}\right) d k \pm U \pm 4 \pi^{2}\left(\frac{b}{2 \pi}\right)^{1 / 2} \frac{e^{2}}{\varepsilon_{\infty} b}
\end{aligned}
$$

The binding energy (BE) given as in [34]

$$
B E=2 E_{p}-E_{b p}
$$

characterises the stability criteria. Here, $E_{p}$ is the single polaron ground state energy in the same approximation. In this case the $\mathrm{BE}$ is given by :

$$
B E=2 E_{p 0}-E_{b p 0}
$$

\section{B. Magnitude of the bangap modulaton}

As the energy difference $E_{b p 0+}-E_{b p 0-}$ denotes the modulated bandgap, it is important to derive it in order to analysise qualitatively the bipolaron effects on the bandgap. The magnitude of the bandgap modulation is defined as [23]:

$$
2 \Delta G=2 G-\left\lfloor\left(E_{b p 0+}\right)_{S O, L O}-\left(E_{b p 0-}\right)_{S O, L O}\right\rfloor
$$

Using Eq. (19) we obtained the magnitude of the bandgap modulation for all coupling regimes and both for LO and SO phonon. From Eq. (15). we observe that $E_{b p 0+}$ and $E_{b p 0_{-}}$are independant of the magnetic field which is differents from other Landau levels energies and others coupling (Eqs. (16) and (17)). So the magnitude of bandgap modulation in weak coupling regime can not be altered by an external magnetic field. This result is similar to the case of polaron $[23,41]$. In others coupling regime (intermediaite and strong), the magnitude of the bandgap is a function of magnetic field.

\section{Absorption coefficient}

The absorption coefficient $\Gamma(\hbar \Omega)$ of the incident light with the energy $\hbar \Omega$ of a free polaron, according to Fermi's golden rule, is $[25,26,35]$ :

$$
\Gamma(\hbar \Omega)=\frac{\pi}{\hbar c n \varepsilon F^{2}} \sum_{f}\left|\left\langle\psi_{0}|V| \psi_{f}\right\rangle\right|^{2} \delta\left(E_{0}+\hbar \Omega-E_{f}\right)
$$

where $n$ is the refractive index of the medium, $c$ equals the speed of light, $\varepsilon$ stands for the permittivity of free space, and $F$ represents the intensity of the electric field vector of the incident photon. $V=e F r$ indicates the time-dependent perturbation, $\psi_{0}$ denotes the ground 
state of a free polaron and $E_{0}$ represents the energy of the ground state. $\psi_{f}$ represents the wave functions of all possible end states with the corresponding energies $E_{f}$. To prevent complicated summation on the final states, a simple model in which the wave functions $\psi_{f}$ of the excited states have been suppressed by the Lee Löw Pines unit transformations have developed [35]. It was noted that Eq. (20). concerns the weak and intermediate coupling regime. Thus, in the weak coupling regime using the same formula we obtain, the absorption coefficient for SO and LO bipolaron as

$$
\Gamma_{w-b p}(\hbar \Omega)=\left\{\begin{array}{l}
\frac{e^{4} \eta}{\hbar^{2} V_{F} c n \varepsilon \varepsilon_{0} m^{2}(\hbar \Omega)^{3}} \sum_{v=1,2} \hbar \omega_{S O, v}\left(\hbar \Omega-\hbar \omega_{S O, v}\right)^{2} \exp \left[\frac{2 z_{0}\left(\hbar \Omega-\hbar \omega_{S O, \nu}\right)}{\hbar V_{F}}\right] \exp \left[-\frac{\left(\hbar \Omega-\hbar \omega_{S O, \nu}\right)}{b V_{F} \hbar}\right] \\
\frac{e^{4} \eta_{0} L_{m} \hbar \omega_{L O}\left(\hbar \Omega-\hbar \omega_{L O}\right)^{2}}{\hbar^{3} V_{F} c n \varepsilon \varepsilon_{0} m^{2}(\hbar \Omega)^{3}}\left[\operatorname{erfc}\left(\frac{\left(\hbar \Omega-\hbar \omega_{L O}\right) \sigma}{2 \hbar V_{F}}\right)\right]^{2} \exp \left[-\frac{\left(\hbar \Omega-\hbar \omega_{L O}\right)}{b V_{F} \hbar}\right]
\end{array}\right.
$$

In the intermediate coupling, the absorption coefficient of SO and LO coefficient is given by:

$$
\Gamma_{\mathrm{int}-b p}(\hbar \Omega)=\left\{\begin{array}{l}
\frac{e^{4} \eta}{\hbar^{2} V_{F} c n \varepsilon \varepsilon_{0} m^{2}(\hbar \Omega)^{3}} \sum_{\nu=1,2} \hbar \omega_{S O, v}\left(\hbar \Omega-\hbar \omega_{S O, v}\right)^{2} \exp \left[\frac{2 z_{0}\left(\hbar \Omega-\hbar \omega_{S O, \nu}\right)}{\hbar \alpha V_{F}}\right] \exp \left[-\frac{\left(\hbar \Omega-\hbar \omega_{S O, v}\right)}{b \alpha V_{F} \hbar}\right] \exp \left[-\left(\frac{1-\alpha}{V_{F} \alpha \hbar}\right)^{2} \frac{\hbar}{m \lambda}\left(\hbar \Omega-\hbar \omega_{S O, \nu}\right)^{2}\right] \\
\frac{e^{4} \eta_{0} L_{m} \hbar \omega_{L O}\left(\hbar \Omega-\hbar \omega_{L O}\right)^{2}}{\hbar^{3} V_{F} c n \varepsilon \varepsilon_{0} m^{2}(\hbar \Omega)^{3}}\left[\operatorname{erfc}\left(\frac{\left(\hbar \Omega-\hbar \omega_{L O}\right) \sigma}{2 \hbar \alpha V_{F}}\right)\right]^{2} \exp \left[-\frac{\left(\hbar \Omega-\hbar \omega_{L O}\right)}{b \alpha V_{F} \hbar}\right] \exp \left[-\left(\frac{1-\alpha}{V_{F} \alpha \hbar}\right)^{2} \frac{\hbar}{m \lambda}\left(\hbar \Omega-\hbar \omega_{L O}\right)^{2}\right]
\end{array}\right.
$$

\section{Results and Discussions}

This section presents the numerical results, we used some constants outlined in Table 1 in our calculations. We assume the Fermi velocity to be equal for all TMDs selected in this paper since it varies slightly for different TMDs [37]. The values of the band gap are adopted in Table 2. For LO phonons, the evaluation of the amounts of band-gap modulation and the relative ratio for the different TMDs are shown in Table 3 and fixed values of $L m=0.5 \mathrm{~nm}, \sigma=0.6 \mathrm{~nm}$ are taken in all TMDs monolayer [42].

Table 1: Parameters of different polar substrates and surfaces optical phonon modes $[41,43]$..

\begin{tabular}{|l|l|l|l|l|l|l|l|}
\hline Quantity (unit) & $h-\mathrm{BN}$ & $\mathrm{SiC}$ & $\mathrm{AiN}$ & $\mathrm{SiO}_{2}$ & $\mathrm{HfO}$ & $\mathrm{ZrO}_{2}$ & $\mathrm{Al}_{2} \mathrm{O}_{3}$ \\
\hline$k_{0}\left(\varepsilon_{0}\right)$ & 5.1 & 9.7 & 9.1 & 3.9 & 22.0 & 24.0 & 12.5 \\
\hline$k_{\infty}\left(\varepsilon_{\infty}\right)$ & 4.1 & 6.5 & 4.8 & 2.5 & 5.0 & 4.0 & 3.2 \\
\hline$\hbar \omega_{S O, 1}(\mathrm{meV})$ & 167 & 146 & 84 & 25 & 101 & 94 & 53 \\
\hline
\end{tabular}




\begin{tabular}{|l|l|l|l|l|l|l|l|}
\hline$\hbar \omega_{S O, 2}(\mathrm{meV})$ & 116 & 60 & 105 & 71 & 196 & 55 & 19 \\
\hline$\eta$ & 0.032 & 0.040 & 0.074 & 0.082 & 0.122 & 0.160 & 0.164 \\
\hline
\end{tabular}

Table 2 The magnitude of bandgap modulation for the different TMDs. The values of intrinsic bandgap are same as from [38,39].

\begin{tabular}{|l|l|l|l|l|}
\hline Quantity (unit) & $\mathrm{MoS}_{2}$ & MoSe $_{2}$ & WS $_{2}$ & WSe $_{2}$ \\
\hline $2 G(m e V)$ & 1870 & 1560 & 2100 & 1650 \\
\hline
\end{tabular}

Table 3 The energies of LO phonons of different TMDs are taken from[25,38].

\begin{tabular}{|l|l|l|l|l|}
\hline Quantity (unit) & $\mathrm{MoS}_{2}$ & $\mathrm{MoSe}_{2}$ & $W S_{2}$ & $\mathrm{WSe}_{2}$ \\
\hline$\hbar \omega_{L O}(m e V)$ & 48 & 34 & 43 & 30 \\
\hline$m\left(m_{0}\right)$ & 0.51 & 0.64 & 0.31 & 0.39 \\
\hline
\end{tabular}

In Fig.2(a). we present the dependences of MBM on the magnetic field for bipolaron in different monolayer TMDs at intermediate coupling regime. It can be seen that the MBM increases with magnetic field. For various TMDs, the MBM are also shown, one can observe that it varies with TMDs monolayer. The most important MBM is obtained with $\mathrm{WS}_{2}$ and the less with $\mathrm{MoSe}_{2}$, thus among the selected TMDs the latter strongly enhances the conductivity. A significant change of MBM relates to each TMDs highlights the impact of the magnetic field on the bandgap modulation in TMDs monolayer.
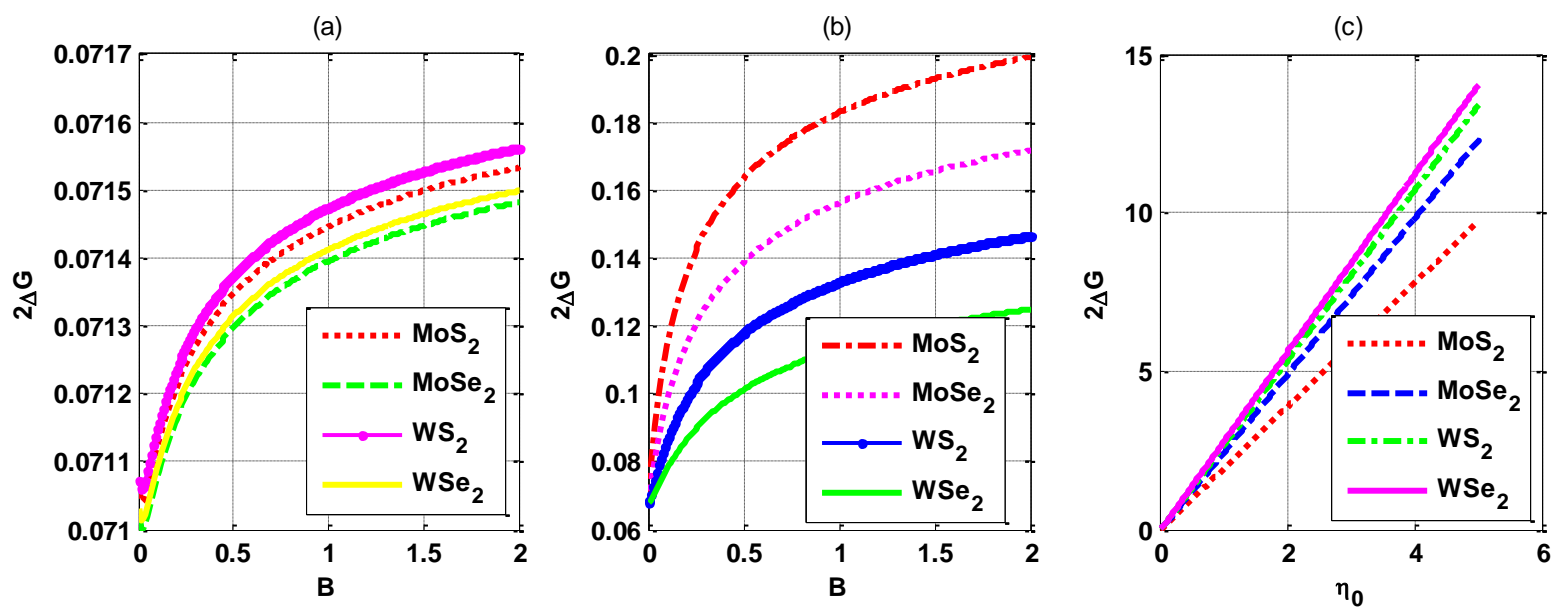

FIG. 2. (a) Magnitude of bandgap modulation versus magnetic field in intermediate coupling regime for different TMDs monolayer on $\mathrm{Al}_{2} \mathrm{O}_{3}$ polar substrates. (b) Magnitude of bandgap 
modulation versus magnetic field in strong coupling regime for differents TMDs monolayer on $\mathrm{Al}_{2} \mathrm{O}_{3}$ polar substrates. (c) Magnitude of bandgap modulation Versus $\eta_{0}$ for different monolayer in weak coupling regime.

Fig.2(b). presents the dependence of $\mathrm{MBM}$ on the magnetic field in different monolayer at strong coupling regime. As in the intermediate regime we can see that MBM increases with magnetic field. We also observe that the MBM varies with TMDs monolayer, then the most important is obtained with $\mathrm{MoS}_{2}$ and the less with $\mathrm{WSe}_{2}$ which is not the case in intermediate coupling regime. Thus the electron phonon coupling affect the MBM for different TMDs monolayer in the presence of bipolaron. Fig. 2(c). presents the MBM versus $\eta_{0}$ for different monolayers in weak coupling regime. One can observed that MBM increases with increasing the coupling parameter $\eta_{0}$. Same result was obtained by [23]. These results show that the bipolaron strongly affects the bandgap of TMDs. Since the coupling parameter characterizes the material, strong coupling favor the modulation of the bandgap. The $\mathrm{WSe}_{2}$ presents the highest MBM. In the investigation of carriers LO phonon coupling in monolayer TMDs, defined a new parameter $g_{f}$ defined by sohier [40] as the coupling strength, which is analogous to the parameter $\eta_{0}$ and pointed out that the 2D Fröhlich coupling is much stronger in TMDs. Moreover, we noticed that the values obtained for the bandgap modulation due to carrier-LO phonon coupling are very close to experimental results $[44,45]$. Then, the LO phonons increase the modulated bandgap then consequently decrease conductivity in TMDs. Among the selected TMDs, the one with highest performance in conductivity is the $\mathrm{MoS}_{2}$.
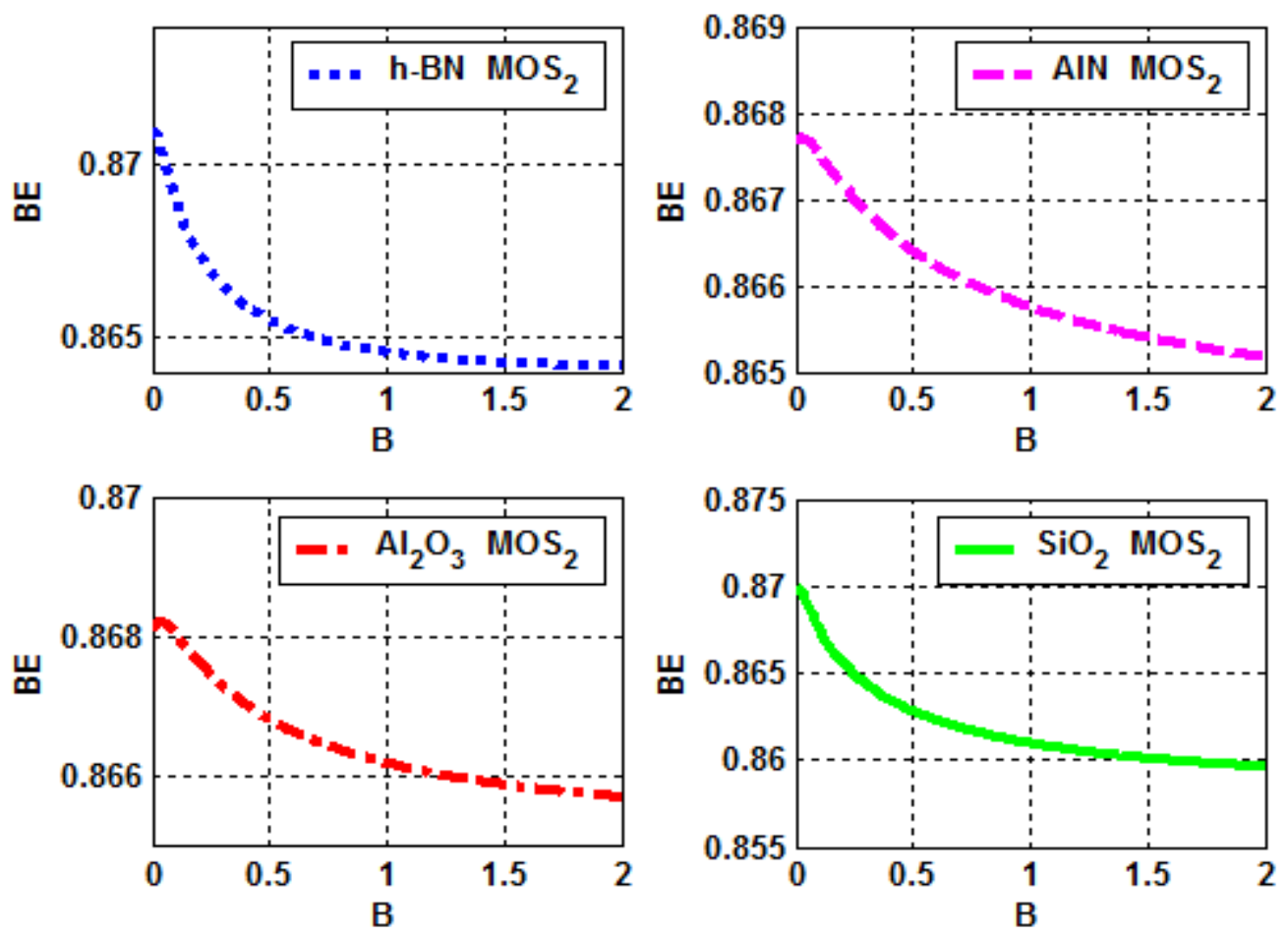
FIG. 3. Binding energy of the bipolaron versus magnetic field $B$ in $\mathrm{MoS}_{2}$ for different polar substrate (intermediate coupling regime)
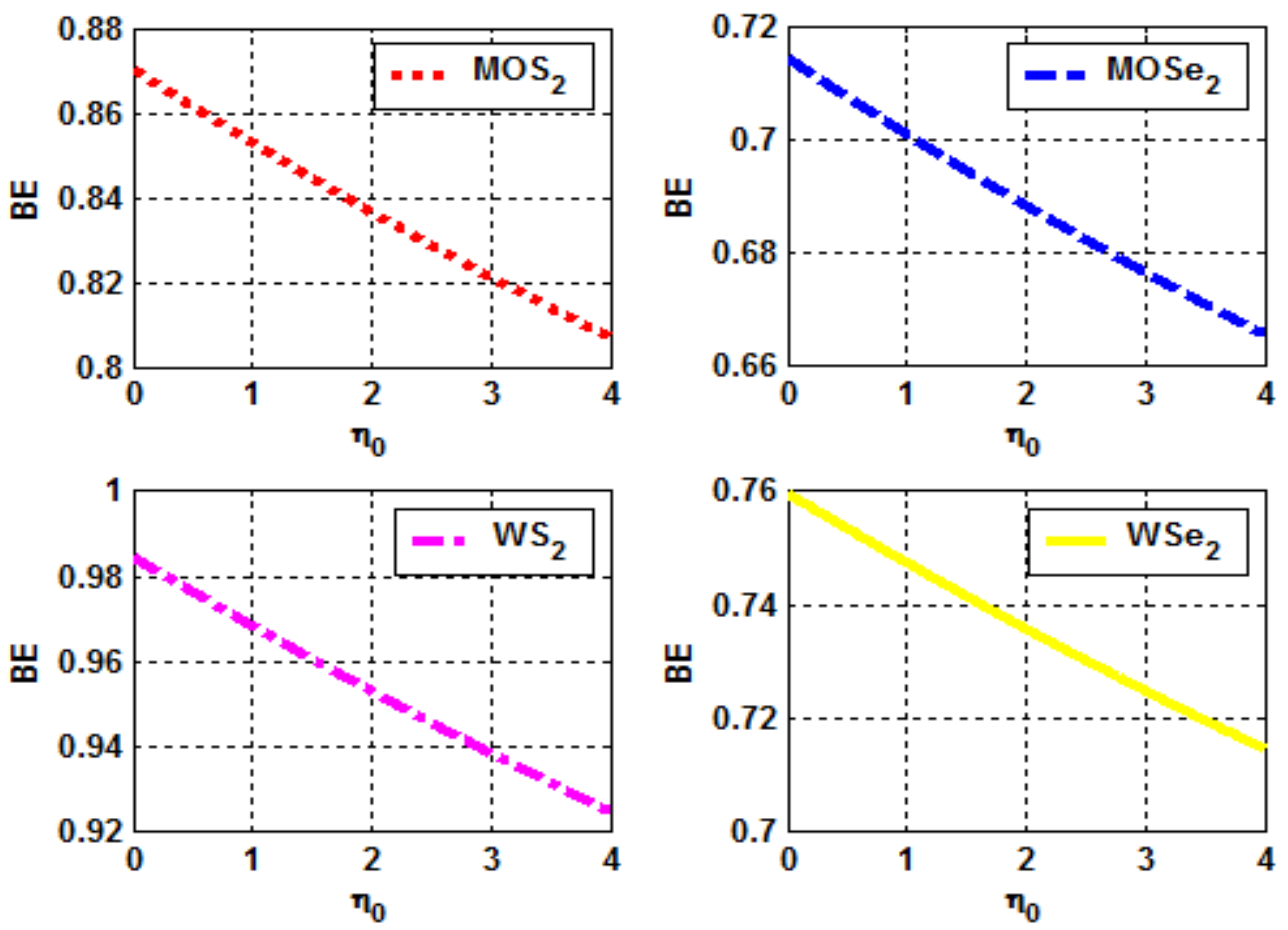

FIG. 4. Binding energy of the bipolaron with parameter $\eta_{0}$ in weak coupling regime for different TMDs monolayer

In Fig.3. the binding energy with magnetic is presented for intermediate coupling regime. One can observed that the binding energy decrease with increasing of magnetic field. The increase of magnetic field enhances the average of coulomb repulsion between the electrons, this result is in accordance with the work of Brosens and Devreese [46]. Thus, despite the enhances of coulomb repulsion, the phonon mediated attractive electron-electron attraction still dominated. That is the reason that the binding energy remain positive and indicated that in all selected TMDs monolayer, the bipolaron is stable. From Fig. 4, the binding energy decreases as $\eta_{0}$ increases, then the bipolaron is stable in the different monolayer TMDs as the binding energy remain positif. 


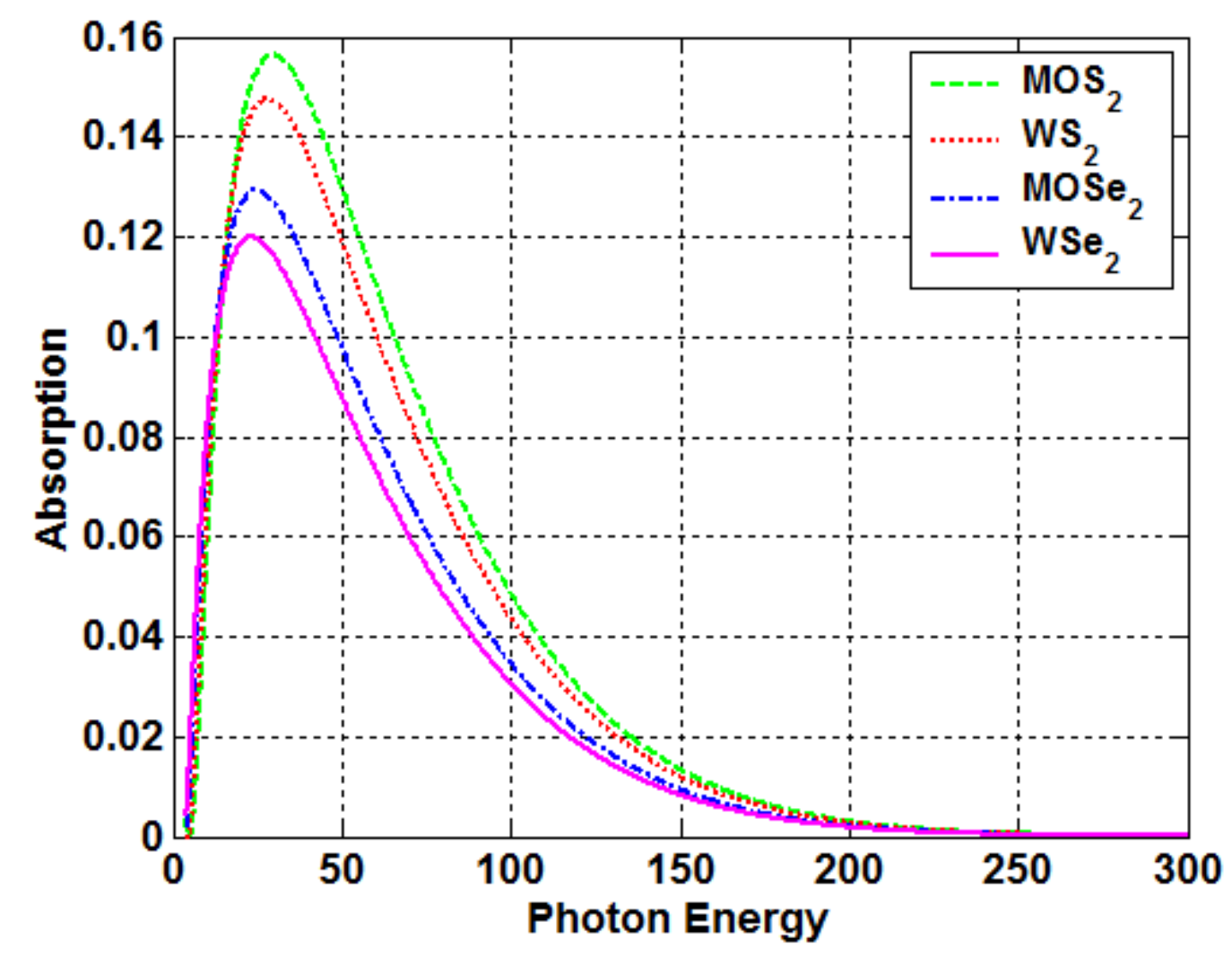

FIG. 5. Optical absorption coefficient versus photon energy in LO phonon in different monolayer TMDS materials for bipolaron in weak coupling regime

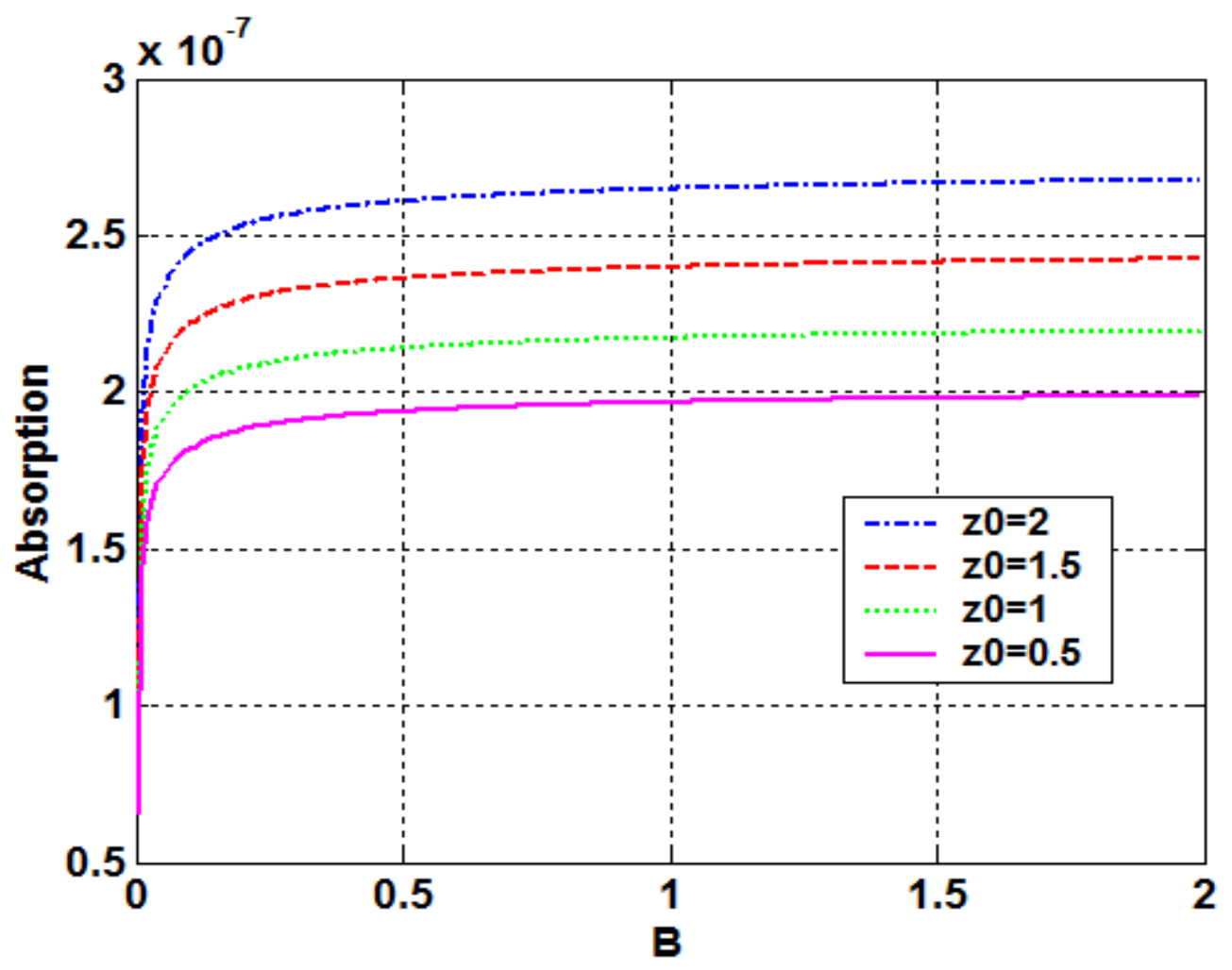


FIG. 6. Optical absorption coefficient (case of SO phonon) versus magnetic field B in $\mathrm{MoS}_{2}$ intermediate coupling regime

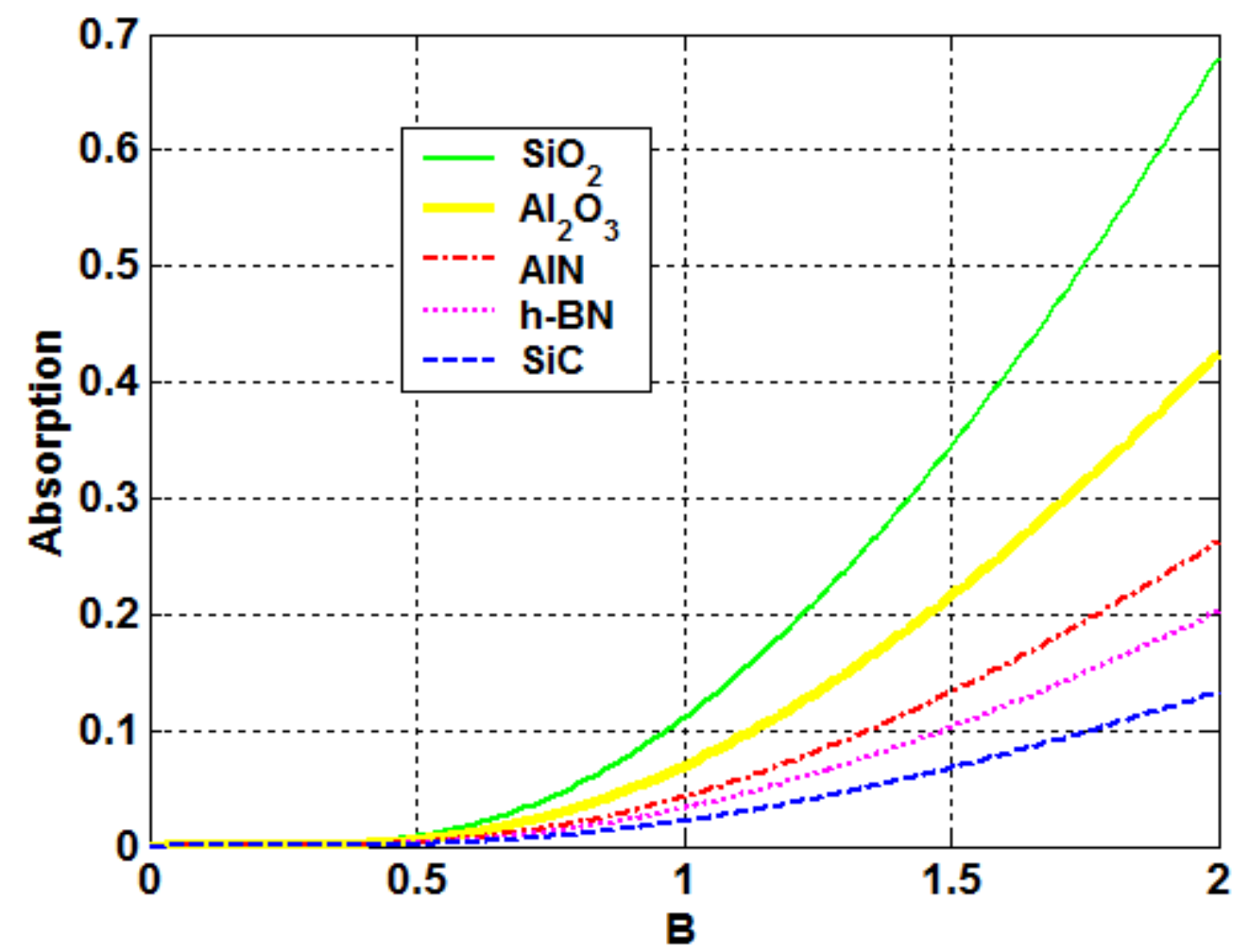

FIG. 7. Optical absorption coefficient (case of SO phonon) versus magnetic field B in $\mathrm{MoS}_{2}$ intermediate coupling regime for different polar substrate

Fig. 5. illustrates the optical absorption of the bipolaron versus the incident photon energy for LO phonons in different layers of TMDs with an weak coupling regime. It can be noted that there is no absorption for $\hbar \Omega \prec \hbar \omega_{L O}$. The threshold value of absorption is at $\hbar \Omega=\hbar \omega_{L O}$. At this value the optical absorption increases and arrives at a maximum and decreases slowly with increasing of photon energy. In fact, these optical absorption behaviours are consistent with previous work on monolayer TMDs and others [26,30,47]. Optical absorption is similar for different TMDs but the strength are not the same for each TMD. This behaviour may be attribuated to the fact that the optical absorption is proportional to the phononic energy of the bipolaron in the different TMDs. This suggests that the lower the phononic energy of the bipolaron in the TMDs, the lower the optical absorption of a bipolaron. A number of past works have been carried out to study the coupling force in such monolayers $[40,42,48]$ Then in $\mathrm{MoS}_{2}$, bipolaron has enough energy to absorb photon than $\mathrm{WSe}_{2}$, this can be due to the dominance of electron-phonon and photon-phonon interations. Comparing the optical absorption in intermediate coupling regime (not shown) with the one in weak coupling regime we observe a 
similar behavior. This can explain why both couplings can be considered identically in some cases.

Fig.6. displays the dependences of optical absorption with magnetic field for the monolayer $\mathrm{MoS}_{2}$ on $\mathrm{SiO}_{2}$ substrate at various values of internal distance between TMDs monolayer and polar substrate. One can observe that the optical absorption is the same for different internal distance when $B=0$, increase slowly and become constant with magnetic field. We also observe that optical absorption increase with internal distance separating the monolayer from polar substrates, proving that the strength of the electron-SO coupling directly depends on the internal distance between the TMDs and the polar substrates. This result is in agreement with that of Li and Wang [30]. In fact, in some studies [49,50], carrier phonon coupling between 2D materials and the polar substrate has also been recognized, where the trends are comparable for the coupling strength with the internal distance. The optical absorption of SO bipolaron at intermediate coupling regime in monolayer $\mathrm{MoS}_{2}$ for different polar substrates is shown in Fig. 7. One can observe the variation of optical absorption with the polar substrates. For $B<0.6$, no absorption is observes in $\mathrm{MoS}_{2}$ then for $B=0.6$ a significant change of absorption relates to each substracts highlights the impact of the magnetic field on the optical absorption in $\mathrm{MoS}_{2}$ monolayer. The greatest optical absorption is observed in $\mathrm{SiO}_{2}$ polar substrate this result is in agreement with those of Hein et al. [51] and lowest for h-BN.

\section{Conclusion}

We theoretically studied optical absorption of bipolaron and it effects on the bandgap modulation in quantum dot of single layer transition metal dichalcogenides under magnetic field for all coupling regime, where both LO and SO phonon are taken into account. Hubrechts method and simple unitary transformation are using. We shown that the MBM of TMDs are flexible. We also found that magnetic field strongly affects the MBM in intermediate and strong coupling regime, thus enhances the conductivity of TMDs monolayer and that in weak coupling regime, the magnetic field cannot be used to tune the MBM. Investigating the optical absorption we found that both the magnetic field and the internal distance separating the monolayer and polar substrates affects the optical absorption in the TMDs. We show that in $\mathrm{MoS}_{2}$, bipolaron has enough energy to absorb than in $\mathrm{WSe}_{2}$. Due to the flexibility of the MBM and the stability of bipolaron in TMD is important to investigate the decoherence of bipolaron in future work.

\section{Conflict of interest:}

The authors declare that they have no conflict of interest. 


\section{References}

[1] R. A. Gordon, D. Yang, E.D. Crozier, D.T. Jiang, R.F. Frindt, Structures of exfoliated single layers of $\mathrm{WS}_{2}, \mathrm{MoS}_{2}$, and $\mathrm{MoSe}_{2}$ in aqueous suspension. Phys. Rev. B 65, 125407 (2002).

[2] W. Choi, N. Chaudhary, G.H. Han, J. Park, D. Akinwande, and Y.H. Lee, Recent development of two-dimensional transition metal dichalcogenides and their applications. Mater. Today 20, 116-130 (2017).

[3] C. Kenfack-Sadem, C.M. Ekengoue, J.E Danga, F.M.C. Fobasso, J.V. Nguepnang, A.J. Fotue, and L.C. Fai, Laser control of magneto-polaron in transition metal dichalcogenides triangular quantum well. Phys. Lett. A 384, 126662 (2020).

[4] K.F. Mak, C. Lee, J. Hone, J. Shan, and T.H. Heinz, Atomically thin $\mathrm{MoS}_{2}$ : a new directgap semiconductor. Phys. Rev. Lett. 105.136805 (2010).

[5] M. Kang, B. Kim, S.H Ryu, S.W. Jung, J. Kim, L. Moreschini, and K.S. Kim, Universal mechanism of band-gap engineering in transition-metal dichalcogenides. Nano. Lett. 17.16101615(2017).

[6] C.S. Lee, G. Jin, S.Y. Seo, J. Kim, C. Han, M.Y. Park, and M.H. Jo, Programmed bandgap modulation within van der Waals semiconductor monolayers by metalorganic vapor-phase epitaxy. Chem. Mater. 32. 5084-5090 (2020).

[7] L. D. Landau, Phys. Z. Sowjetunion 3. 644-645 (1993).

[8] B. S. Kandemir D. Akay, The effect of electron-phonon coupling in spin-orbit-coupled graphene. Philosophical Magazine, 97. 2225-2235 (2017).

[9] M.F.C Fobasso, C. Kenfack-Sadem, E. Baloitcha, A.J. Fotué, and L.C. Fai, Lifetime and dynamics of polaron and bipolaron in graphene nanoribbon under laser. The European Physical Journal Plus, 135. 1-18 (2020).

[10] D. Emin, Barrier to recombination of oppositely charged large polarons. J. Appl. Phys., 123. 055105 (2018).

[11] W. Xin-Ran, S. Yi, and Z. Rong, Field-effect transistors based on two-dimensional materials for logic applications. Chineese Physics B, 22. 098505 (2013).

[12] B. Liu, W. Niu, X. Ruan, C. Zhu, X. Wang, L. He, and Y. Xu, Observation of Small Polaron and Acoustic Phonon Coupling in Ultrathin La0. 7Sr0. 3MnO3/SrTiO3 Structures. physica status solidi (RRL)-Rapid Research Letters, 13. 1800657(2019).

[13] Z. Yin, H. Li, L. Jiang, Y. Shi, Y. Sun, G. Lu, Q. Zhang, X. Chen, and H. Zhang, Singlelayer $\mathrm{MoS}_{2}$ phototransistors. ACS. Nano. 6. 74-80 (2012). 
[14] H.S. Lee, S.W. Min, Y.G. Chang, M.K. Park, T. Nam, H. Kim, J.H. Kim, S. Ryu, S. and S. Im, $\mathrm{MoS}_{2}$ nanosheet phototransistors with thickness-modulated optical energy gap. Nano. Lett. 12. 3695-3700 (2012).

[15] Z. Ni, Q. Liu, K. Tang, J. Zheng, J. Zhou, R. Qin,... and J. Lu, Tunable bandgap in silicene and germanene. Nano. Lett. 12. 113-118 (2012).

[16] J. Lee, J. Huang, B.G Sumpter, and M. Yoon, Strain-engineered optoelectronic properties of 2D transition metal dichalcogenide lateral heterostructures. 2D. Mater. 4. 021016 (2017).

[17] K.S. Thygesen, Calculating excitons, plasmons, and quasiparticles in 2D materials and van der Waals heterostructures. 2D. Mater. 4. 022004 (2017).

[18] M.M. Ugeda, A.J. Bradley, S.F. Shi, F.H. da Jornada, Y. Zhang, D.Y. Qiu, W. Ruan, S.K. Mo, Z. Hussain, Z.X. Shen, F. Wang, S.G. Louie, and M.F. Crommie, Giant bandgap renormalization and excitonic effects in a monolayer transition metal dichalcogenide semiconductor. Nat. Mater. 13. 1091-1095 (2014).

[19] B. Liu, W. Zhao, Z. Ding, I. Verzhbitskiy, L. Li, J. Lu, and K.P. Loh, Engineering Bandgaps of Monolayer $\mathrm{MoS}_{2}$ and $\mathrm{WS}_{2}$ on Fluoropolymer Substrates by Electrostatically Tuned Many- Body Effects. Adv. Mater. 28. 6457-6464 (2016).

[20] A. Raja, A. Chaves, J. Yu, G. Arefe, H.M. Hill, A.F. Rigosi,..... and C. Nuckolls, Coulomb engineering of the bandgap and excitons in two-dimensional materials. Nat. Comm. 8. 1-7 (2017).

[21] F. Withers, O. Del Pozo-Zamudio, A. Mishchenko, A.P. Rooney, A. Gholinia, K. Watanabe,... and K.S. Novoselov, Light-emitting diodes by band-structure engineering in van der Waals heterostructures. Nat. Mater.14. 301-306 (2015).

[22] M.L.Trolle, T.G. Pedersen, and V. Véniard, Model dielectric function for 2D semiconductors including substrate screening. Sci. Rep. 7. 39844 (2017).

[23] Y. Xiao, Z.Q. Li, and Z.W. Wang, Polaron effect on the bandgap modulation in monolayer transition metal dichalcogenides. J. Phys.: Cond. Matter. 29. 485001 (2017).

[24] J.T. Devreese, Fröhlich polarons from OD to 3D: concepts and recent developments. J. Phys.: Cond. Mater. 19. 255201 (2007).

[25] J.T. Devreese, and S.A. Alexandre, Fröhlich polaron and bipolaron: recent developments. Rep. Prog. Phys. 72. 066501 (2009).

[26] J.T. Devreese, Fröhlich Polarons. Lecture course including detailed theoretical derivations. arXiv preprint arXiv:1611.06122 (2016).

[27] M. Koschorreck, D. Pertot, E. Vogt, B. Fröhlich, M. Feld, and M. Köhl, Attractive and repulsive Fermi polarons in two dimensions. Nature 485. 619-622 (2012). 
[28] S.N. Klimin, and J.T. Devreese, Optical conductivity of a strong-coupling Fröhlich polaron. Phys. Rev. B 89. 035201 (2014).

[29] H. Sezen, H. Shang, F. Bebensee, C. Yang, M. Buchholz, A. Nefedov, ... and C. Wöll, Evidence for photogenerated intermediate hole polarons in $\mathrm{ZnO}$. Nat. Comm. 6. 1-5 (2015).

[30] P-F. Li, and Z-W. Wang, Optical absorption of Fröhlich polaron in monolayer transition metal dichalcogenides. J. Appl. Phys. 123. 204308 (2018).

[31] C.M. Chow, H. Yu, A.M. Jones, J. Yan, D.G. Mandrus, T. Taniguchi,... and X. Xu,. Unusual exciton-phonon interactions at van der Waals engineered interfaces. Nano. Lett. 17. 1194-1199 (2017).

[32] C. Chen, J. Avila, S. Wang, Y. Wang, M. Mucha-Kruczyński, C. Shen,.., ... and M.C. Asensio, Emergence of Frohlich polaron from interlayer electron-phonon coupling in van der Waals heterostructure. arXiv preprint arXiv:1707.00184 (2017).

[33] G-B. Liu, H. Pang, Y. Yao, and W. Yao, Intervalley coupling by quantum dot confinement potentials in monolayer transition metal dichalcogenides. News J. Phys. 16. 105011 (2014).

[34] Y-H. Ruan, and Q-H. Chen, Ground and excited states of bipolarons in two and three dimensions. Comm. Theor. Phys. 48.169 (2007).

[35] J.T. Devreese, W. Huybrechts, and L. Lemmeks, On the optical absorption of free polarons at weak coupling. Physica Status Solidi (b) 48. 77-86 (1971).

[36] O.L.Berman, and Y.K. Roman, Superfluidity of dipolar excitons in a transition metal dichalcogenide double layer. Phys. Rev. B 96. 094502 (2017).

[37] A. Kormányos, G. Burkard, M. Gmitra, J. Fabian, V. Zólyomi, N.D. Drummond, and V. Fal'ko, k. p theory for two-dimensional transition metal dichalcogenide semiconductors. 2D. Mater. 2. 022001 (2015).

[38] K.K. Kaasbjerg, S. Thygesen, and K.W. Jacobsen, Phonon-limited mobility in n-type single-layer $\mathrm{MoS}_{2}$ from first principles. Phys. Rev. B 85. 115317 (2012).

[39] A. Kuc, N. Zibouche, and T. Heine, Influence of quantum confinement on the electronic structure of the transition metal sulfide TS $_{2}$. Phys. Rev. B 83. 245213 (2011).

[40] T. Sohier, M. Calandra, and F. Mauri, Two-dimensional Fröhlich interaction in transitionmetal dichalcogenide monolayers: Theoretical modeling and first-principles calculations. Phys. Rev. B 94. 085415 (2016).

[41] Z-W. Wang, L. Lei, and Z-Q Li, Energy gap induced by the surface optical polaron in graphene on polar substrates. Appl. Phys. Lett. 106. 101601 (2015).

[42] A. Thilagam, Exciton formation assisted by longitudinal optical phonons in monolayer transition metal dichalcogenides. J. Appl. Phys. 120. 124306 (2016). 
[43] I-T. Lin, and J-M. Liu, Surface polar optical phonon scattering of carriers in graphene on various substrates. Appl. Phys. Lett. 103. 081606 (2013).

[44] S. Tongay, J. Zhou, C. Ataca, K. Lo, T.S. Matthews, J. Li, J.C. Grossman, and J. Wu, Thermally driven crossover from indirect toward direct bandgap in 2D semiconductors: $\mathrm{MoSe}_{2}$ versus $\mathrm{MoS}_{2}$. Nano. Lett. 12. 5576-5580 (2012).

[45] A. Arora, K. Nogajewski, M. Molas, M. Koperski, and M. Potemski, Exciton band structure in layered $\mathrm{MoSe}_{2}$ : from a monolayer to the bulk limit. Nanoscale 7. 20769-20775 (2015).

[46] F. Brosens, J.T. Devreese, Stability of bipolarons in the presence of a magnetic field. Phys. Rev. B 54. 9792 (1996).

[47] T. Sohier, M. Gibertini, M. Calandra, F. Mauri, and N. Marzari, Breakdown of optical phonons' splitting in two-dimensional materials. Nano. Lett. 17. 3758-3763 (2017).

[48] K.K. Kaasbjerg, K.S. Bhargavi, and S.S. Kubakaddi, Hot-electron cooling by acoustic and optical phonons in monolayers of $\mathrm{MoS}_{2}$ and other transition-metal dichalcogenides. Phys. Rev. B 90. 165436 (2014).

[49] J. Ryou, Y.S. Kim, S. Kc, and K. Cho, Monolayer $\mathrm{MoS}_{2}$ bandgap modulation by dielectric environments and tunable bandgap transistors. Sci. Rep. 6. 1-8 (2016).

[50] C. Chen, J. Avila, S. Wang, Y. Wang, C. Shen, R. Yang, B. Nosarzewski, T.P. Devereaux, G. Zhang, and M.C. Asensio, Emergence of interfacial polarons from electron-phonon coupling in graphene/h-BN van der waals heterostructures. Nano. Lett. 18. 1082-1087 (2018).

[51] N.D. Hien, C.V. Nguyen, N.N. Hieu, S.S. Kubakaddi, C.A. Duque, M.E. Mora-Ramos,., ... and H.V. Phuc, Magneto-optical transport properties of monolayer transition metal dichalcogenides. Phys. Rev. B 101. 045424 (2020). 